\title{
Efecto morfológico y térmico de filmes flexibles de etilen vinil alcohol reforzados con óxido de grafeno
}

\section{Morphological and thermal effects of ethylene vinyl alcohol film reinforced with graphene oxide}

Lourdes Marcela Yataco-Lazaro ${ }^{1}$, Jesús González-Ruiz",3, Sueli Virginio ${ }^{4}$, Rene R. Oliveira ${ }^{4}$, Wilson S. Maia ${ }^{5}$, Francisco Valenzuela-Díaz ${ }^{5}$, Esperidiana Moura ${ }^{4}$,

1 University of São Paulo, Faculty of Pharmaceutical Sciences, Av. Professor Lineu Prestes, 580, São Paulo, PA 05508-00, Brazil

2 Biomaterials Center, University of Havana, Av. Universidad / G y Ronda, PA 10400, Havana, Cuba 3 High Polytechnic Institute "José Antonio Echeverría", Street 114 / 119 and 127, PA 10800, Havana, Cuba

${ }^{4}$ Nuclear and Energy Research Institute - IPEN-CNEN/SP Av. Prof. L. Prestes, 2242 São Paulo, PA 05508000, Brazil

${ }^{5}$ Metallurgical and Materials Engineering Department, Polytechnic School, University of São Paulo, Av. Prof. Mello de Moraes, 2463, São Paulo, PA 05508-030, Brazil

DOI: https://doi.org/10.33017/RevECIPeru2015.0017/

\section{Resumen}

Se estudió los efectos morfológicos y térmicos de los filmes de EVOH reforzados con 0.1 y 0.5 wt. \% de GO. El $\mathrm{EVOH}$ ha sido ampliamente usado en embalajes para alimentos porque preserva la calidad de los productos debido a su excelente desempeño de barrera de gas y su buena transparencia. El GO fue preparado vía oxidación química del grafito y fue exfoliado en nanoláminas usando el método sonoquímico. Los filmes fueron preparados por extrusión en estado fundido y extrusión por soplado, luego fueron caracterizados por análisis de XRD, TG y SEM, la correlación entre sus propiedades fue discutida.

Descriptores: etilen vinil alcohol, óxido de grafeno, filmes flexibles, propiedades mecánicas, propiedades térmicas.

\section{Abstract}

This work studied the morphological and thermal effects of ethylene vinyl alcohol (EVOH) film reinforced with 0.1 and 0.5 wt. \% of graphene oxide (GO). The EVOH has been widely used in packaging for food to preserve the quality of the products due to its excellent gas barrier performance and its good transparency. The GO was prepared via chemical oxidation of graphite and was exfoliated into nanosheets using the sonochemical method. The films were prepared by melt and blown extrusion, then were characterized by XRD, TG and SEM analysis, the correlation between properties was discussed.

Keywords: ethylene vinyl alcohol, graphene oxide, flexible films, mechanical properties, thermal properties.

\section{Introducción}

Los embalajes para alimentos requieren un excelente desempeño de barrera de gas y humedad para preservar su calidad durante su almacenamiento y manipulación con el fin de incrementar el tiempo de vida media del producto final. Entre los polímeros más usados para este propósito tenemos el polímero termoplástico Etilen 
Vinil Alcohol (EVOH). El EVOH presenta una excelente resistencia química, así como una muy buena propiedad de barrera de gases como el oxígeno, solventes orgánicos y aromas de los alimentos, además de una muy buena transparencia [1-3].

En estos últimos años, la adición del grafeno en filmes poliméricos está siendo intensamente estudiada porque mejoraría la impermeabilidad y las propiedades mecánicas y térmicas del filme [1, 3-6].

Por otra parte, esas mejoras son frecuentemente observadas adicionando bajos contenidos de óxido de grafeno, debido a su gran área de superficie $[4,7]$. Se ha reportado que con la adición del 0.7 wt. \% de óxido de grafeno en nanocompósitos de polivinil alcohol se observó un aumento del $76 \%$ en la resistencia a la tracción y un $62 \%$ en el módulo de Young [8]. Además, los nanocompósitos conteniendo 0.9 wt. \% de óxido de grafeno en una matriz de poliestireno mostró mejoras significativas en las propiedades mecánicas (aumento del $57 \%$ en el módulo elástico y un $70 \%$ en la resistencia) [9]. También, en un estudio reciente se mostró que la adición de grafeno o óxido de grafeno en una matriz polimérica de EVOH puede mejorar sus propiedades mecánicas y su permeabilidad al oxígeno en las películas de $\mathrm{EVOH}$ [6, 10-12]. Sin embargo, para conseguir mejoras significativas en las propiedades de los nanocompósitos, el óxido de grafeno debería ser exfoliado y bien dispersado en la matriz polimérica [13].

La oxidación química del grafito es el método más usado para la preparación de grafeno [14]. El óxido de grafeno (GO) es obtenido empleando ácidos concentrados (ácido sulfúrico, ácido nítrico y ácido fosfórico) y agentes altamente oxidantes (permanganato de potasio o perclorato de potasio) [14-15]. Este método fue desarrollado por J. Hummers et al. [16] y fue modificado por los diferentes investigadores para mejorar su eficiencia. La síntesis de GO usando una variante mejorada del método de J. Hummers et al. fueron reportados por D. Marcano et al. [17]. Las ventajas de esta variante son el empleo de un protocolo relativamente simple con temperatura controlada a $50^{\circ} \mathrm{C}$ y una emisión de gases no tóxicos durante la preparación, razón por la cual sería adecuado para la preparación de GO a escala industrial. Sin embargo, dicho método tiene la desventaja de que la proporción de conversión de grafito a GO no es de un $100 \%$.
Recientemente, N. Huang et al. reportaron una nueva variante que simplifica el proceso de síntesis de GO $[14,18]$. Este método tiene la ventaja de obtener cerca del $100 \%$ de conversión de grafito a GO y permite además obtener nanoláminas de GO.

Sin embargo, para el uso de materiales compósitos, esto representa una desventaja, puesto que las nanoláminas presentan dimensiones a escala micrométrica. Por otro lado, la exfoliación de GO puede ser desarrollado por agitación y comúnmente por sonicación en solventes [14, 19, 20]. En este estudio, GO fue preparado por el método de J. Hummers et al. Además, las partículas obtenidas fueron usadas en la elaboración de los compósitos de películas flexible de EVOH/ GO.

\section{Preparación y caracterización del GO y las películas de EVOH/ GO.}

\subsection{Preparación de GO.}

El GO fue obtenido a partir de las escamas de grafito (Quimesp Química Ltda.) empleando el método usado por N. Huang et al. (método simplificado de J. Hummers et al.) [14]. Luego el GO (en solución acuosa) fue sonicado a temperatura ambiente, usando un homogeneizador ultrasónico de alta intensidad a $19 \mathrm{kHz}$ de frecuencia ultrasónica y 500 Watts de potencia ultrasónica (Unique, modelo DES500).

La técnica utilizada para la obtención y caracterización de GO se encuentra detallada en nuestro trabajo "Effects of graphene oxide addition on mechanical and thermal properties of EVOH films" [21].

\subsection{Preparación de los filmes de EVOH.}

Las películas de EVOH/GO fueron preparados por extrusión en estado fundido, usando una extrusora de doble tornillo Haake Rheomex P332, L/D 25. Los materiales que salen de la extrusora fueron enfriados para mejorar su estabilidad dimensional, granulados, secados una vez más y por último sometidos a un proceso de extrusión por soplado para obtener las muestras de películas. El proceso utilizado para la obtención de los filmes de EVOH/GO se encuentra detallado en nuestro trabajo

"Effects of graphene oxide addition on mechanical and thermal properties of evoh films" [21]. 


\subsubsection{Caracterización del GO y los filmes de EVOH/GO.}

Difracción de Rayos-X: Los análisis de DRX son comunmente usados para estudiar la estructura de los filmes y la adecuada intercalación de la masa fundida del polímero y/o la nanocarga en los filmes. Los análisis de DRX fueron realizados empleando un difractómetro de rayos-X (Rigaku Denki Co. Ltd., modelo Multiflex), operando bajo las siguientes condiciones: radiación CuKa $(\lambda=1.5406 \AA), 40 \mathrm{kV} \mathrm{y}$ $20 \mathrm{~mA}$, con este procedimiento, los ángulos (2Ө) de difracción de todas las muestras fueron caracterizadas de $2^{\circ}$ a $50^{\circ}$. Estos análisis fueron empleados para demostrar que el $\mathrm{GO}$ ha sido intercalado en la matriz de EVOH.

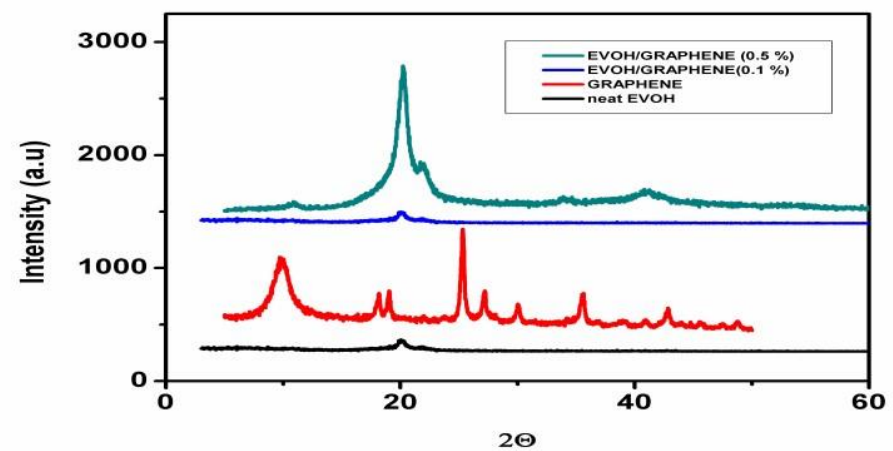

Figura 1. Difractograma del grafeno, EVOH y los filmes de EVOH/GO.

Análisis Termogravimétrico (TG): Los análisis de TG fueron realizados empleando una termobalanza (Mettler Toledo, modelo TGA/SDTA851e), operando bajo las siguientes condiciones: atmósfera de oxígeno a $50 \mathrm{~mL} / \mathrm{min}$ con una razón de calentamiento de $10^{\circ} \mathrm{C} \mathrm{min}^{-1}$ en el intervalo de temperatura entre 25 y $500^{\circ} \mathrm{C}$. Estos análisis fueron empleados para determinar las características térmicas de los filmes de EVOH puro y EVOH/ GO.

Tabla 1. Temperatura de degradación y pérdida de masa de los filmes flexibles de EVOH y filmes de EVOH/GO.

\begin{tabular}{|c|c|c|c|}
\hline \multirow[b]{2}{*}{ Filmes } & $\overline{T_{i}(c)}$ & $T_{\max (d)}$ & \multirow{2}{*}{\begin{tabular}{|l|} 
Pérdida \\
de Masa
\end{tabular}} \\
\hline & (ㅇ) & (으) & \\
\hline & & & (\%) \\
\hline 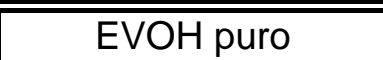 & 356.5 & $\overline{385.9}$ & 97.5 \\
\hline \multirow{2}{*}{$\underset{\text { (a) }}{E V O H / G O}(0.1$ wt. \%) } & 345.9 & 371.0 & 99.6 \\
\hline & 355.2 & 379.4 & \\
\hline
\end{tabular}
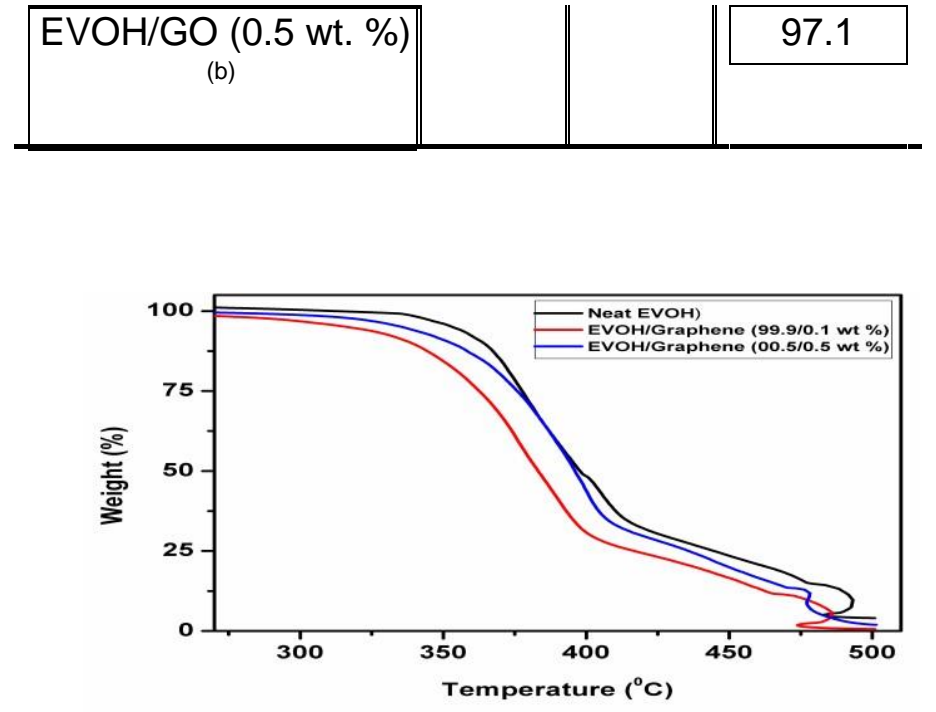

(a) EVOH/GO (99/0.1 wt \%);
${ }^{\text {(b) } E V O H / G O}(99.5 / 0.5$ wt \%);
(c) Temperatura inicial de pérdida de massa; ${ }^{(d)}$ Temperatura
máxima de pérdida de massa.

Figura 2. Curvas termogravimétricas para el EVOH y los filmes de EVOH/GO.

Microscopía Electrónica de Barrido (MEB): Los análisis de MEB fueron realizados empleando un microscopio electrónico de barrido (Phillips, modelo LX-30), las muestras fueron criofracturadas en nitrógeno líquido y luego las superficies fracturadas fueron revestidas con una fina capa de oro $y$ posteriormente observadas en el MEB.

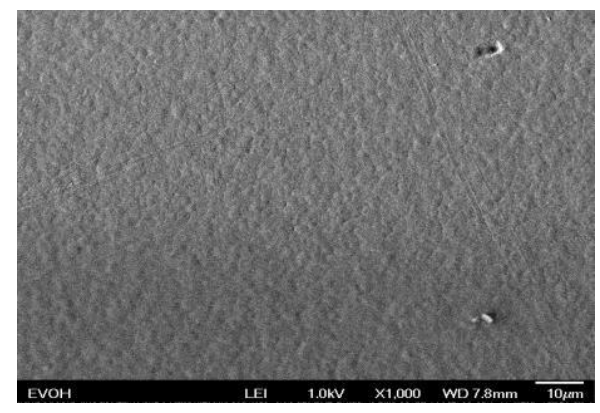

Figura 3. Micrografía por microscopía electrónica de barrido para el filme de EVOH.

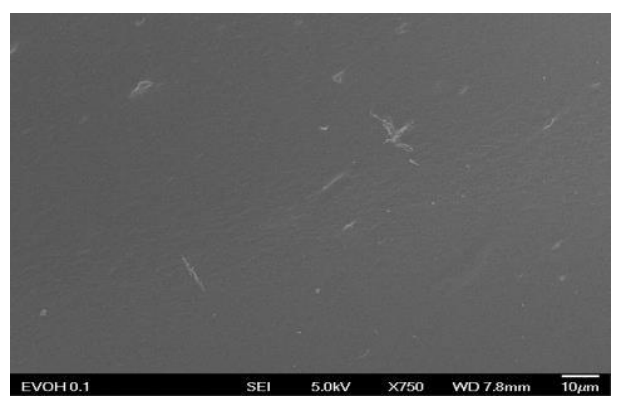


Figura 4. Micrografía por microscopía electrónica de barrido para el filme de EVOH/ 0.1 wt.\% de GO.

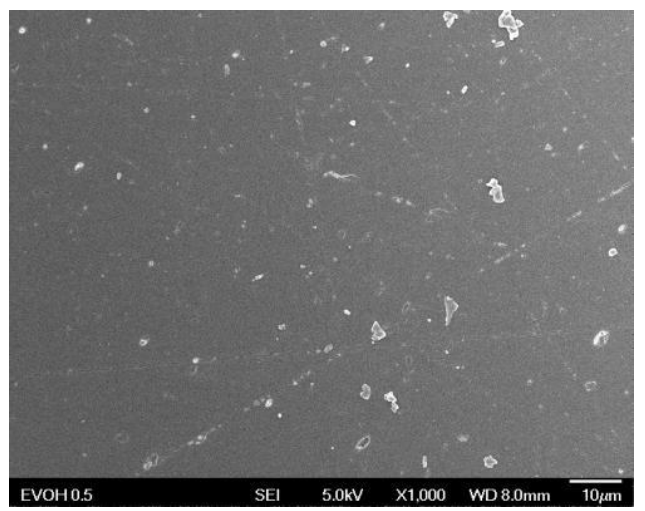

Figura 5. Micrografía por microscopía electrónica de barrido para el filme de EVOH/ 0.5 wt. \% de GO.

\section{Resultados y discusión}

Con la técnica de DRX determinamos el tamaño y la distribución del GO. Entre cada capa de grafito existe una distancia c, cuando se comienza a realizar la oxidación, esta distancia entre las capas debería aumentar debido a la exfoliación de las capas del grafito. El valor del desplazamiento observado en el difractograma nos indica que efectivamente la distancia entre capas ha aumentado, y por medio de la ecuación de la Ley de Bragg, podemos obtener la expansión en la dirección $\mathrm{c}$ de la estructura hexagonal del grafito.

Como se puede observar en la figura 1, el difractograma de DRX del GO presenta ondas difractadas correspondientes a $2 \theta=25.0^{\circ}$ a $26.0^{\circ}$ y $2 \Theta=18^{\circ}$, Kim et al. indica valores próximos a $26.4^{\circ}$ (espacio de $0.34 \mathrm{~nm}$ ) y al rango de $14.1^{\circ}$ a $14.9^{\circ}$ para la identificación del grafito puro y GO, respectivamente [1], lo cual sugiere que no se obtuvo el $100 \%$ de conversión de grafito a GO. Mientras tanto en el difractograma de los filmes de $\mathrm{EVOH} / 0,1$ wt. \% de GO no se observa ninguna onda difractada característica del grafito puro, lo que implica que el GO al 0,1 wt. \% fue altamente intercalado debido al mayor número de grupos funcionales que contienen oxígenos voluminosos formado a través de un proceso de oxidación más intenso, además, en su micrografía del SEM no hubo ni un rastro de láminas apiladas de grafito puro. En el difractograma de los filmes de $\mathrm{EVOH} / 0,5$ wt. \% de

GO se observa algunas ondas difractadas características del grafito puro más anchas pero con menos intensidad, lo cual indicaría que algo de grafito, mantiene su estado original de grafito puro y otra pequeña cantidad de grafito que fue ligeramente intercalada con la matriz de EVOH [1].

La estructura morfológica de los filmes de EVOH reforzados con GO (previamente sintetizado y exfoliado) fue examinada usando la técnica del MEB. La figura 3 muestra la micrografía del filme de $\mathrm{EVOH}$ puro, se puede observar una superficie ligeramente fracturada, relativamente homogénea y áspera, lo cual sugiere que los filmes de EVOH presenta una estructura dúctil, lo cual estaría conforme con lo indicado por la literatura [1]. La figura 4 muestra la micrografía del filme de $\mathrm{EVOH} / 0.1$ wt. \% de GO, se puede observar las escasas partículas de GO dispersados e interactuando con la matriz de $\mathrm{EVOH}$, mientras que la figura 5 muestra la micrografía del filme de $\mathrm{EVOH} / 0.5$ wt. \% de GO, se puede observar pequeñas partículas transparente de GO dispersadas homogéneamente en gran parte de la superficie, sin embargo también presenta algunas partículas monolaminares más transparente de mayor tamaño, lo cual sugiere que dichas partículas podría ser $\mathrm{GO}$ en sus inicios de exfoliación. Tanto en la figura 4 y 5 , la adición del GO en la matriz del EVOH soluciona el problema de la superficie fracturada presente en el filme de EVOH.

En la figura 2, se observa la curva de TG y en la tabla 1 se evalúa el comportamiento de descomposición de las muestras. Todos los materiales mostraron una pérdida de masa de $98.5 \% \pm 1 \%$. El filme de $\mathrm{EVOH}$ puro fue descompuesto en dos pasos, el primer paso a $356.5^{\circ} \mathrm{C}$ y el segundo paso a $385.9{ }^{\circ} \mathrm{C}$. La incorporación de GO a la matriz de $\mathrm{EVOH}$ disminuyó ligeramente la temperatura del primer paso $\left(345.9^{\circ} \mathrm{C}\right.$ y $355.2^{\circ} \mathrm{C}$ para 0.1 y 0.5 wt. $\%$ de $\mathrm{GO}$, respectivamente), mientras tanto la temperatura del segundo paso también disminuyó ligeramente (371.0 ${ }^{\circ} \mathrm{C}$ y $379.4^{\circ} \mathrm{C}$ para 0.1 y 0.5 wt. \% de GO, respectivamente), esto sugiere que los filmes de $\mathrm{EVOH} / \mathrm{GO}$ son térmicamente tan estable como el filme de EVOH puro.

\section{Conclusiones}

Tanto los resultados del anterior y presente estudio que hemos realizado muestra que la adición del GO, promete modificaciones significativas en las principales propiedades morfológicas, mecánicas y térmicas de los filmes de EVOH, además la síntesis y la exfoliación del GO fueron realizadas satisfactoriamente. Los resultados muestran que el GO ha sido exitosamente intercalado con la matriz de 
EVOH para la obtención de filmes flexibles para su aplicación en embalajes para alimentos para que preserve la calidad de los productos debido a su excelente desempeño de barrera de gas y su buena transparencia.

\section{Agradecimientos}

Los autores desean expresar sus sinceros agradecimientos a las siguientes instituciones: IPENUSP, IP-USP y la Universidad de la Habana por la ayuda en la conducción de los análisis.

\section{Referencias}

[1] S. W. Kim, H. M. Choi. High Performance Polymers. 27 (2014) 694-704.

[2] D. Kim, H. Kwon, J. Seo. Polymer Composites. 35 (2014) 644-654.

[3] H. M. Kim, H. S. Lee. Carbon Letters. 15 (2014) 50-56.

[4] T. K. Das, S. Prusty. Polymer-Plastics Technology and Engineering. 52 (2013) 319331.

[5] J. R. Potts, D. R. Dreyer, C. W. Bielawski. Polymer. 52 (2011) 5-25

[6] W. Alkarmo, J. Thomassin, C. Macosko, M. Trifkovic, C. Detrembleur, C. Jérôme. (Paper presented at the Belgian Polymer Group (BPG) Annual Meeting, Houffalize, Belgium, 18 May 2015).

[7] O. M. Istrate, J. N. Coleman. Carbon. 78 (2014) 243-249.

[8] D. E. Kranbuehl, H. C. Schniepp, "Polyamide composites containing graphene oxide sheets," US Patent 20150114472, 2014.

[9] T. Ramanathan, A. A. Abdala, S. Stankovich, D. A. Dikin, M. Herrera-Alonso, R. D. Piner, D. H. Adamson, H. C. Schniepp, X. Chen, R. S. Ruoff, S. T. Nguyen, I. A. Aksay, R. K. Prud'Homme, L. C. Brinson. Nature nanotechnology. 3 (2008) 327-331.

[11] J. Liang, Y. Huang, L. Zhang, Y. Wang, Y. Ma, T. Guo, Y. Chen. Advanced Functional Materials. 19 (2009) 2297-2302.

[12] J. Yang, L. Bai, G. Feng, X. Yang, M. Lv, Ch.

Zhang, H. Hu, X. Wang. Industrial \& Engineering Chemistry Research. 52 (2013) 16745-16754.

[13] E. T. Thostenson, C. Li , T. W Chou. Composites Science and Technology. 65 (2005) 491-516.
[14] N. M. Huang, H. N. Lim, C. H. Chia, M. A. Yarmo, M. R. Muhamad. International Journal of Nanomedicine. 6 (2011) 3443-3448.

[15] Y. Zhu, S. Murali, W. Cai, X. Li, J. W. Suk, J. R. Potts, R. S. Ruoff. Advanced Materials. 22 (2010) 3906-3924.

[16] J. Hummers, S. William, Offeman, E. Richard. "Preparation of graphitic acid," US Patent 2798878, 1957.

[17] D.C. Marcano, D. V. Kosynkin, J. M. Berlin, A. Sinitskii, Z. Sun, A. Slesarev, L. B. Alemany, W. Lu, J. M. Tour. ACS Nano, 4 (2010) 4806-4814.

[18] X. Huang, X. Qi, F. Boey, H. Zhang. Chemical Society Reviews. 41 (2012) 666686.

[19] K. Krishnamoorthy, G.S Kim, S.J Kim. Ultrasonics Sonochemistry. 20 (2013) 644649.

[20] X. Qi, T. Zhou, S. Deng, G. Zong, X. Yao, Q. Fu. Journal of Materials Science. 49 (2014). 17851793.

[21] J. González-Ruiz, L. Yataco-Lazaro, S. Virginio, M. G. Silva-Valenzuela, E. Moura, F. Valenzuela-Díaz. Effects of graphene oxide addition on mechanical and thermal properties of evoh films. Aprobado para publicación en: Characterization of Minerals, Metals, and Materials 2016. ed. Hoboken : John Wiley \& Sons, Inc., 2016. 